\title{
Identification of key genes and crucial modules associated with coronary artery disease by bioinformatics analysis
}

\author{
XUEMEI ZHANG ${ }^{1}$, XIAOSHU CHENG ${ }^{2}$, HUIFENG LIU ${ }^{3}$, CHUNHUA ZHENG $^{1}$, \\ KUNRUI RAO ${ }^{1}$, YI FANG ${ }^{1}$, HAIRONG ZHOU ${ }^{4}$ and SHENGHE XIONG ${ }^{1}$ \\ ${ }^{1}$ Department of Cardiology, Third Affiliated Hospital of Nanchang University; \\ ${ }^{2}$ Department of Cardiology, Second Affiliated Hospital of Nanchang University, Nanchang, Jiangxi 330008; \\ ${ }^{3}$ Department of Cardiology, Xiaolan People's Hospital, Zhongshan, Guangdong 528415; \\ ${ }^{4}$ Department of Cardiology, Second People's Hospital, Mudanjiang, Heilongjiang 157000, P.R. China
}

Received December 19, 2013; Accepted May 28, 2014

DOI: $10.3892 /$ ijmm.2014.1817

\begin{abstract}
The aim of this study was to identify key genes associated with coronary artery disease (CAD) and to explore the related signaling pathways. Gene expression profiles of $110 \mathrm{CAD}$ and 112 non-CAD, healthy patients [CAD index $(\mathrm{CADi})>23$ and $=0$, respectively] were downloaded from the Gene Expression Omnibus (GEO) database (accession: GSE12288). The differentially expressed genes (DEGs) in CAD were identified using t-tests, and protein-protein interaction (PPI) networks for these DEGs were constructed using the Search Tool for the Retrieval of InteractiNg Genes (STRING) database. The Database for Annotation, Visualization and Integrated Discovery (DAVID) tool was used to identify potentially enriched biological processes (BP) among the DEGs using Gene Ontology (GO) terms, and to identify the related pathways using the Kyoto Encyclopedia of Genes and Genomes (KEGG) pathway database. In addition, expressionactivated subnetworks (crucial modules) of the constructed PPI networks were identified using the jActiveModule plugin, and their topological properties were analyzed using NetworkAnalyzer, both available from Cytoscape. The patient specimens were classified as grade I, II and III based on CADi values. There were 151 DEGs in grade I, 362 in grade II and 425 in grade III. In the PPI network, the gene GRB2, encoding the growth factor receptor-bound protein 2 , was the only common DEG among the three grades. In addition, 10 crucial modules were identified in the PPIs, 4 of which showed significant enrichment for GO BP terms. In the 12 nodes with the highest betweenness centrality, we found two genes,
\end{abstract}

Correspondence to: Dr Xiaoshu Cheng, Department of Cardiology, Second Affiliated Hospital of Nanchang University, Minde Road 1, Nanchang 330008, P.R. China

E-mail: chengxiaoshu123@hotmail.com

Key words: coronary artery disease, differentially expressed genes, protein-protein interaction, topological properties, pathway analysis encoding GRB2 and the heat shock $70 \mathrm{kDa}$ protein 8 (HSPA8). Moreover, the chemokine and focal adhesion signaling pathways were selected based on their relative abundance in CAD. The GRB2 and HSPA8 proteins, as well as the chemokine and focal adhension signaling pathways, might therefore be critical for the development of CAD.

\section{Introduction}

Coronary artery disease (CAD), also called coronary arteriosclerosis, is the most common type of heart disease (1). It is the leading cause of death in the United States in both genders $(2,3)$. CAD occurs when the coronary arteries which supply blood to the heart muscle become partially blocked or clogged, which then leads to indurative and stenotic coronary arteries (4). As with the development of atherosclerosis, reduced amounts of blood and oxygen are transferred to the heart muscles, resulting in angina or a heart attack (5). Most heart attacks occur when a blood clot suddenly cuts off the blood supply to the heart, causing permanent heart damage. Over time, CAD can also weaken the function of heart muscles and contribute to heart failure and arrhythmias (6).

Survival following coronary artery bypass graft surgery (CABG) and medical therapy in patients with CAD has been studied in both randomized trials and observational treatment comparisons (7-11). A number of randomized trials have shown that treatment with percutaneous coronary intervention (PCI) or mineral technologies (MT) is associated with higher rates of angina and subsequent revascularization compared to CABG treatment, but no significant difference has been found in mortality or rates of myocardial infarction (MI) between these types of treatment (12). Despite the lack of concluding evidence from randomized trials and prospective observational comparisons, the application of coronary angioplasty (PTCA) has increased dramatically over the past decade (13). Current pharmacologic therapy for CAD patients presents multiple limitations, such as compliance issues and medication side-effects. For revascularization procedures, there is often a need to repeat the procedures, which is accompanied by organ damage (14). Therefore, the development of new methods for the treatment of CAD is necessary. 
Gene therapy offers such an attractive solution. It has the potential to locally and continuously provide therapeutic proteins at the disease site, and can potentially lead to reversal of the pathophysiology associated with the disease. Therefore, in order to identify key genes and related signaling pathways associated with CAD, which could potentially serve in gene therapy, we performed a meta-analysis on the GSE12288 CAD gene expression profiling dataset, downloaded from the Gene Expression Omnibus (GEO) database. Differentially expressed genes (DEGs) and related protein-protein interaction (PPI) networks were identified, along with expression-activated subnetworks (crucial modules) of the PPI networks, enriched biological functions and the related signaling pathways.

\section{Materials and methods}

Data source. The gene expression dataset GSE12288 (15) was downloaded from the GEO database (16). The 222 patients assessed in this dataset were divided into two groups based on their respective CAD index (CADi): the control group, with 112 healthy individuals $(\mathrm{CADi}=0)$, and the case group, with 110 patients (CADi $>23$ ). Samples from all individuals had been hybridized on the Affymetrix Human Genome U133A (HG-U133A) array on a GPL96 platform (Affymetrix, Santa Clara, CA, USA).

DEG identification. The GSE12288 dataset had been normalized using MAS5 (Affymetrix) (15). Log (base 2) transformation of the downloaded raw data was performed with the GEOquery module, available in R (17). CAD groups comprising case samples with the same CADi were defined. Subsequently, a between-subjects t-test was performed to identify DEGs of each CAD group using the T-test module of MultiExperiment Viewer (18) and a significance threshold $\mathrm{P}<0.01$ compared to the control group. For the pairwise alignment of genes differentially expressed between two CAD groups (dataset A and B), the Jaccard similarity coefficient or index was computed according to the following formula:

$$
J(A, B)=\frac{|A \cap B|}{|A \cup B|}
$$

Based on the proximity of DEGs as defined by the Jaccard index value, the case samples were reclassified in three CAD grades (Fig. 1). Subsequently, re-comparison between CAD grade samples and those of the control group was performed, and the DEGs were identified with a significance threshold $\mathrm{P}<0.01$.

Construction of PPI. It was shown that functionally associated genes are co-expressed, with their gene expression levels varying depending on the cell type and state (19). Thus, the Search Tool for the Retrieval of InteractiNg Genes (STRING) (http://string-db.org/) database (20) was used to build PPI networks for the identified DEGs, so as to predict their interactions. A combination score of $>0.3$ was used as the threshold. The topological properties of the resulting PPI network were visualized and analyzed with Cytoscape (21). We used the jActiveModule plug-in of Cytoscape to further identify the significant modules in the network as previously described $(22,23)$.

Enrichment analysis of significant modules. The Database for Annotation, Visualization and Integrated Discovery (DAVID) provides a comprehensive set of functional annotation tools for biological interpretation of large gene lists. DAVID was used here to group the functions of DEGs in modules, identify enriched biological processes, and identify the pathways associated with the DEGs in the most significant modules. Function and pathway terms were retrieved from the Gene Ontology (GO) and the Kyoto Encyclopedia of Genes and Genomes (KEGG) databases, respectively $(24,25)$.

Topological analysis of PPI networks. The topological properties of the PPI networks were analyzed using NetworkAnalyzer available in Cytoscape (26). NetworkAnalyzer allows to compute and display a comprehensive set of topological parameters, such as the number of nodes and edges, the betweenness and the stress centrality. Betweenness centrality, a measure of the centrality of a node in a network, is the fraction of shortest paths between node pairs that pass through the node of interest (27). The stress centrality is the number of shortest paths a node lies on (28).

\section{Results}

Identification of DEGs. All control samples were collected as group A. Based on their CADi values (23, 32, 37, 42, 48, $56,63,67,74,82$ and 100), the case samples were grouped in CAD groups, each comprising samples of similar CADi. The CADi $=56$ group comprised only one sample, while all other CAD groups comprised $>1$ samples. Following identification of genes differentially expressed in each CAD group compared to the control group, pairwise alignment of the genes differentially expressed between two CAD groups was conducted. Then, the case groups were reclassified into three grades based on their similarity degree: grade I comprised samples with CADi $=23-42$; grade II with CADi $=48-67$; and grade III, with CADi $=74-100$. Finally, we identified the differentially expressed genes, defined as DEGs of each grade, through a new comparison between each grade and the control group. There were 151 DEGs in grade I, 362 in grade II and 425 in grade III.

PPI networks and functional enrichment analysis. PPIs of DEGs in grade I, II and III were constructed by calculating confidence values using the STRING tool (29). In the resulting networks (Fig. 2), genes that simultaneously belonged to more than two grades were few, and only the gene encoding growth factor receptor-bound protein 2 (GRB2) was common to grades I, II and III. The expression values of the DEGs and the p-values from t-test comparisons were used to identify the expression activated subnetworks (crucial modules) of the PPI network using the jActiveModule in Cytoscape. Table I displays the 10 modules identified with this procedure, 4 of which were significantly enriched for biological processes (colored in Fig. 3). Module 4 genes belonged to grade I samples, module 2 and 3 belonged to grade II and module 1 belonged to grade III. 


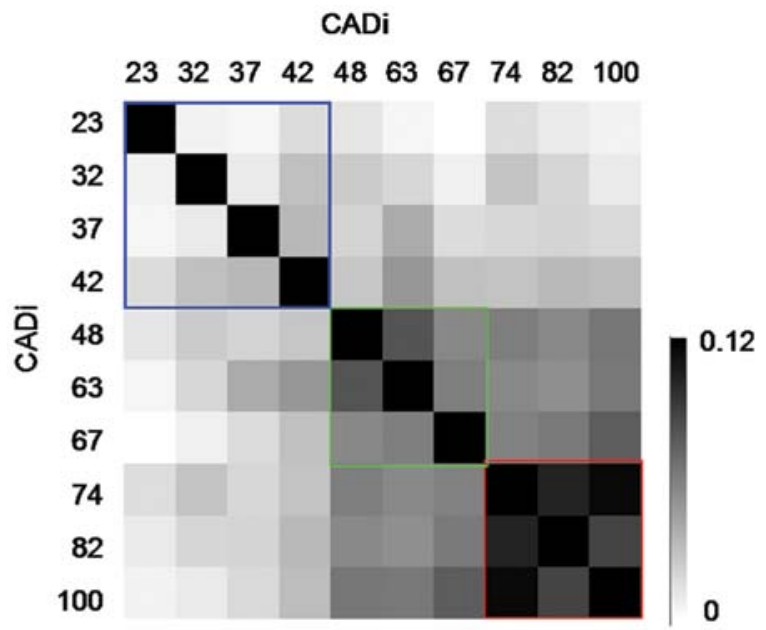

Figure 1. Jaccard index matrix. Blue box, grade I samples with coronary artery disease index $(\mathrm{CADi})=23-42 ;$ green box, grade II samples with $\mathrm{CADi}=48-67 ;$ and red box, grade III with CADi $=74-100$. The deeper the color is, the higher the Jaccard index is, i.e., the higher the similarity.

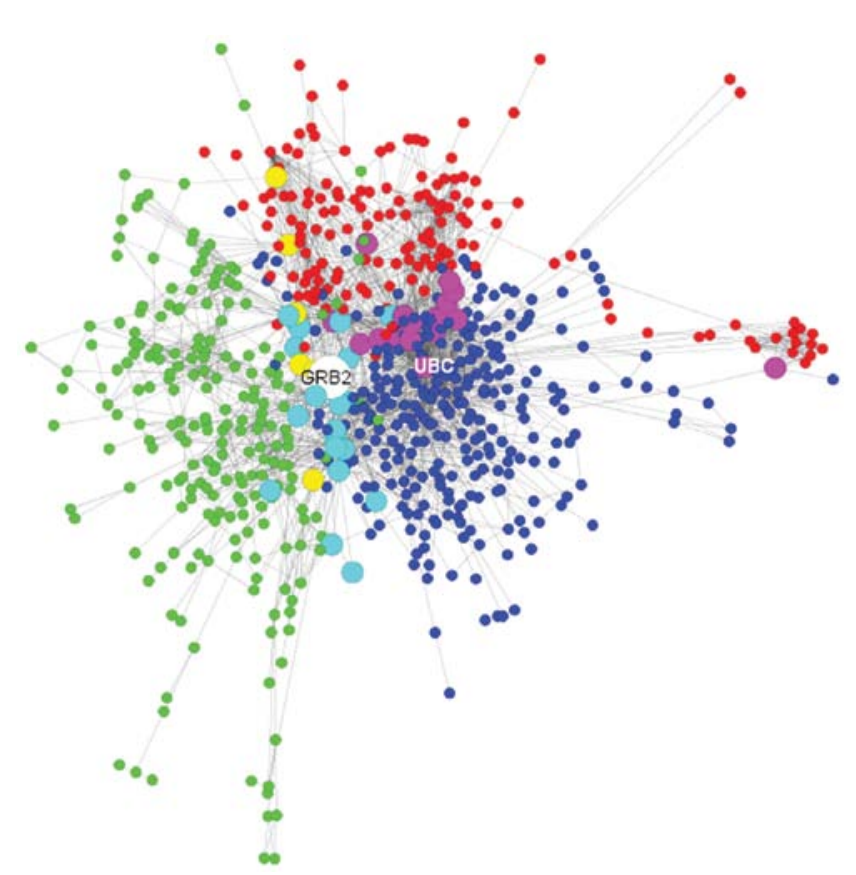

Figure 2. Protein-protein interaction (PPI) network of grades I, II and III. Red dots, differentially expressed genes (DEGs) in coronary artery disease (CAD) grade I samples; green dots, DEGs in grade II; blue dots, DEGs in grade III; yellow dots, DEGs common to grade I and II; cyan blots DEGs shared between grades II and III; magenta dots, DEGs shared between grades I and III; white dot, DEG identified in all three grades. The size of each dot indicates the grade of the gene.

KEGG pathway analysis. To gain further insights into the function of DEGs belonging to the crucial modules, we used the online biological classification tool DAVID to retrieve functional annotations from $\mathrm{GO}$ (biological process terms) and KEGG for the DEGs and perform an enrichment analysis for the GO terms. Table II lists the enriched biological processes in which the DEGs belonging to the crucial modules are involved. The crucial modules 1-4 were related to cell apoptosis $(\mathrm{P}=2.70 \mathrm{E}-03)$, protein catabolic process $(\mathrm{P}=1.40 \mathrm{E}-04)$, cell signaling $(\mathrm{P}=2.30 \mathrm{E}-03)$ and telomere maintenance $(1.70 \mathrm{E}-06)$,

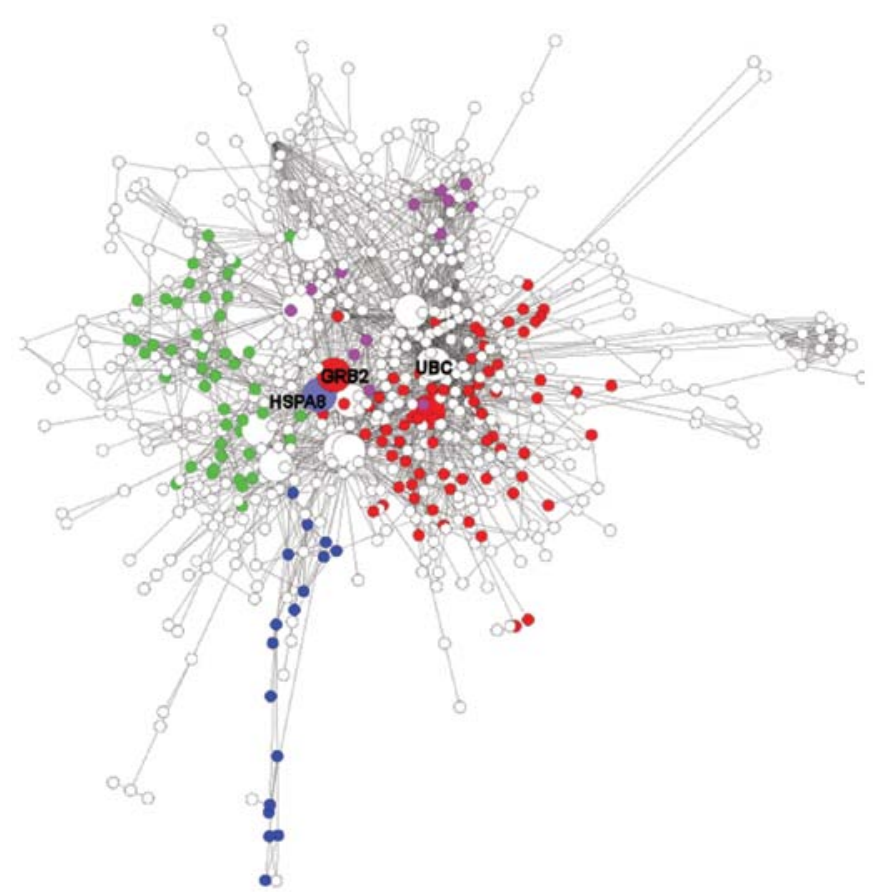

Figure 3. Four crucial modules identified with jActiveModule further showing significant enrichment for biological processes. Red dots, module 1; green dots, module 2; blue dots, module 3; magenta dots, module 4; purple dot, gene shared between modules 1 and 2 . The size of each dot indicates the degree of betweenness centrality.

respectively. In addition, according to the percentage of identified DEGs in each pathway relative to the total number of DEGs, which is defined as their relative abundance (RA), we ranked the KEGG pathways in the selected modules. Figure 4 shows the top 10 pathways, in which DEGs from the 4 modules are involved. These include the chemokine signaling pathway ( $\mathrm{RA}=0.176)$, focal adhension $(\mathrm{RA}=0.143)$, and regulation of actin $(\mathrm{RA}=0.099)$. The chemokine pathway and related genes as depicted in the KEGG Pathway database are shown in Fig. 5.

Topological analysis of PPI networks. PPI topology analysis showed that the node with the higher betweenness centrality (the biggest dots in Figs. 3 and 6) was the node comprising the gene $U B C$, with betweenness centrality $\sim 0.42$. The stress centrality of this node was $\sim 10^{6}$ (Fig. 7), a high level in all nodes. In the PPI network, $U B C$ was shown to be commonly shared between grades I and III (Fig. 2), but did not belong to any crucial modules (Table I, Fig. 3). Among the 12 nodes showing the highest betweenness centrality, only GRB2 and HSPA8 appeared in crucial modules.

\section{Discussion}

In this study, in order to identify the key genes associated with CAD and the related signaling pathways, so as to ultimately gain knowledge on the disease that would be applicable to early diagnosis and therapy, we identified 151 DEGs in samples from grade I patients, 362 genes from grade II and 425 genes from grade III patients. The number of selected DEGs increased along with the rise in CADi values, and 


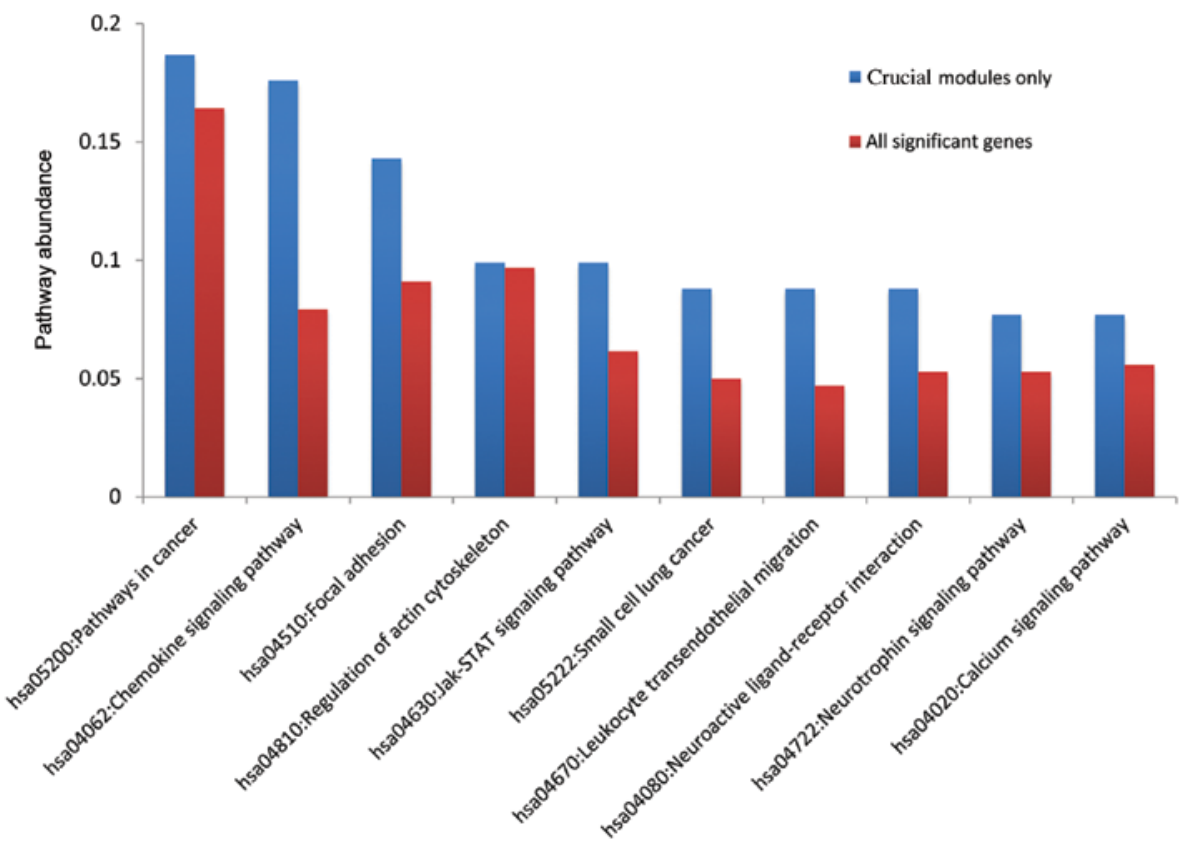

Figure 4. The top 10 Kyoto Encyclopedia of Genes and Genomes (KEGG) pathways in the modules, selected by jActiveModule.

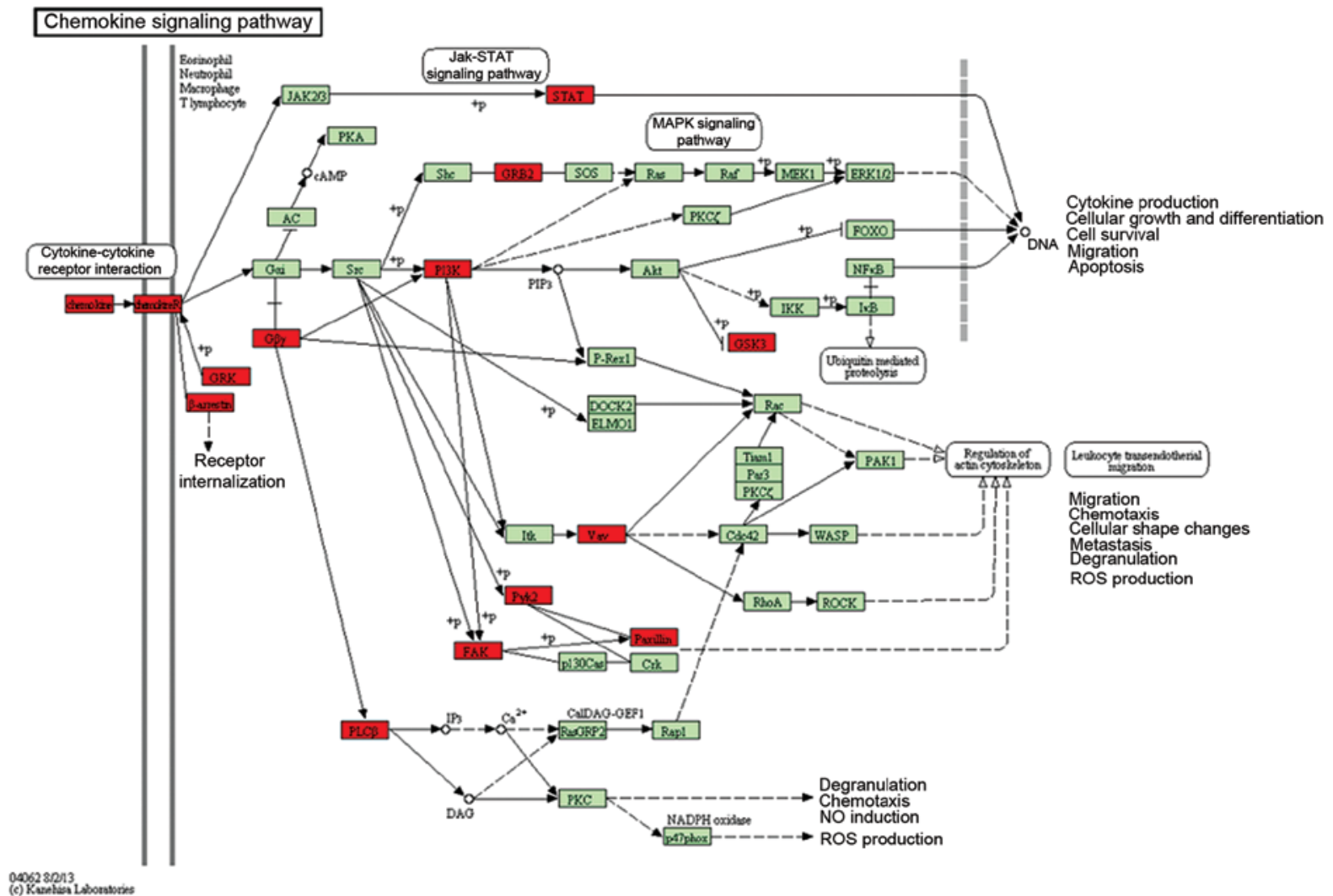

Figure 5. Chemokine signaling pathway. Red boxes denote differentially expressed genes (DEGs), and green boxes the non-DEGs.

there was only one overlapping gene (GRB2) among the three grades. To explore the potential molecular interactions among the DEGs, PPI networks were constructed. Analysis of their topological properties indicated that GRB2 and HSPA8 genes, showing the highest betweenness centrality, belong to crucial modules of the PPI network. The protein product of GRB2, Grb2, is widely distributed in various tissues and cells and participates in numerous biological functions $(30,31)$. The docking proteins of the Grb2-associated binder family (Gab1, Gab2 and Gab3), serving as important signaling components, may amplify and integrate signal transduction pathways in response to various stimuli, such as cytokines (32). In addition, as a molecular switch, Grb2 is a ubiquitously expressed linker protein that couples growth factor receptor activation to down- 
Table I. The 10 crucial modules and the differentially expressed genes (DEGs).

\begin{tabular}{|c|c|c|}
\hline Module & Score & Gene list \\
\hline 1 & 6.0996 & $\begin{array}{l}\text { ALAD, MAP2K7, PPARD, PTPRT, EEA1, HSPA8, ARRB2, CD68, HSPE1, TMEM11, GRM3, PPM1A, } \\
\text { CCL15, RPS25, PYCRL, HSPD1, CBLB, ATAD3A, IL22, GLUL, FLT4, CHD1, TTC3, BCHE, MGMT, } \\
\text { CTNND1, CAPN5, PPP2R4, HIST1H2AJ, PDE6A, CCDC72, PXN, EIF3M, CABIN1, SUV420H1, SSB, } \\
\text { PTK2B, GRK1, DNAJA3, REV3L, PKM2, GRB2, PITPNM1, TGM4, CETP, STK11, HDAC5, CALM3, } \\
\text { IGHV1-69, MRPL19, ALB, HIST2H4A, HIST2H4B, IGHA1, VAV1, ACAN, DAP, LEPR, CS, GYPC, } \\
\text { CCL14, C22orf28, IGHM, DNAJB2, ARHGDIA, RPS27A, HIST1H2BH, HSPA5, S1PR4, CCR1, PTK2, } \\
\text { CD6, SDC2, IGHG1, CCR7, SMARCB1, CDKN1C, IARS, FANCA, STAT1, NCAPH, CTNNB1, HSPA8, } \\
\text { COPS6, NIN, GATA1, PSMF1, FANCC, FANCF, USP14, DARS2, CTNND1, TES, SKP2, PTRF, CDH11, } \\
\text { RBX1, FBXO46 }\end{array}$ \\
\hline 2 & 5.8049 & $\begin{array}{l}\text { S1PR1, DACT1, ACP1,AXIN1, YWHAQ, ISG20, COPS5, SP110, IRF9, FGF2, BUB1B, UBE2L6, } \\
\text { SON, PSMB10, OAS3, STAT3, REXO2, ANKRD6, NASP, EED, GSK3A, IGFBP1, PTPRD, IGF2, EPHB2, } \\
\text { BTRC, NUF2 SERPINA5, MS4A4A, ITGA2B, GPR183, TBX1, ITGAV }\end{array}$ \\
\hline 3 & 3.7185 & AMELY, C1QB, LAMB3, FGL2, NPPA, IGFBP1, ITGB8, C1QA, LAMB4, FGA, COL4A4 \\
\hline 4 & 2.1734 & PTGIS, ZNF3, NDRG2, ATP1B1 \\
\hline 5 & 2.0949 & SLC39A14, CKMT1A, SLC39A2, SLC6A8, CKMT1B \\
\hline 6 & 1.7816 & CCDC106, TP53 \\
\hline 7 & 1.7670 & CHP, ITPR1, PLCB1, EDNRB, F2RL1, PIK3R1, PTAFR, LPAR1, CBLB, GNG5 \\
\hline 8 & 1.7518 & TERF1, VAV1, GRAP2, BRCA1, NBN, PTPN11, TERF2, EED, XRCC5, MPZL1, LIG4, SUZ12, SIAH2 \\
\hline 9 & 1.6902 & MCM6, SMARCC1, PPARG, L3MBTL1, NFYB, HOXA10, NCAPH, TP53, VDR, NCOR1, BAG1, MGMT \\
\hline 10 & 1.6280 & MEIS 1 \\
\hline
\end{tabular}

Table II. Modules with enriched biological processes as defined by Gene Ontology (GO) terms.

\begin{tabular}{lll}
\hline Module & \multicolumn{1}{c}{ Enriched biological process } & P-value \\
\hline 1 & Regulation of caspase activity & $5.90 \mathrm{E}-04$ \\
& Programmed cell death & $2.70 \mathrm{E}-03$ \\
2 & Protein catabolic process & $1.40 \mathrm{E}-04$ \\
& Cellular response to hormone stimulus & $8.90 \mathrm{E}-04$ \\
& Positive regulation of protein modification process & $3.40 \mathrm{E}-04$ \\
3 & Integrin-mediated signaling pathway & $2.30 \mathrm{E}-03$ \\
& Telomere maintenance & $1.70 \mathrm{E}-06$ \\
& Double-strand break repair & $2.00 \mathrm{E}-05$ \\
\hline
\end{tabular}

The analysis was performed with the Database for Annotation, Visualization and Integrated Discovery (DAVID) tool.

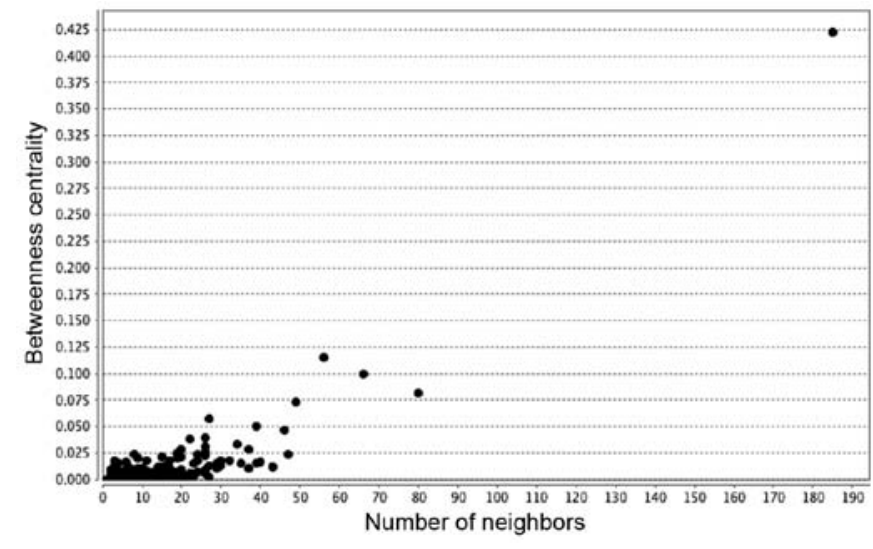

Figure 6. Nodes with the highest betweenness centrality. The size of each dot indicates the degree of betweenness centrality.

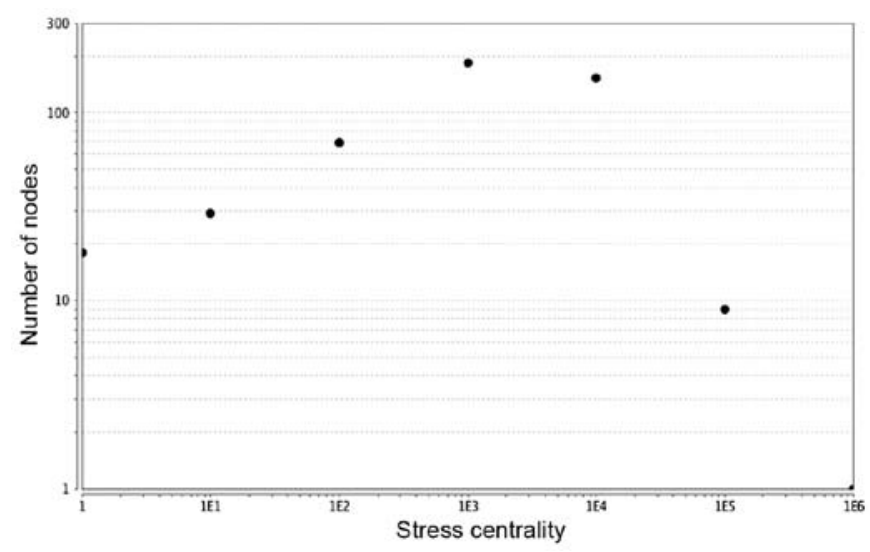

Figure 7. Stress centrality. 
stream mitogen-activated protein kinases (MAPK), including the c-Jun N-terminal kinase (JNK2), the extracellular-signalregulated kinase (ERK) and p38 MAPK $(33,34)$, which may play a role in cardiac hypertrophy, atherosclerosis and vascular restenosis, suggesting that Grb2 might be a pharmacological therapeutic target for CAD (35). The HSPA8 protein (also known as HSP73 or HSC70) displays 86\% amino acid similarity with the heat-inducible protein of the same family HSPA1A (36). Constitutively expressed HSPA8 is involved in protecting cardiac muscle cells from injuries such as oxidative challenge $(37,38)$. Moreover, heritable variations in the HSPA8 gene might increase the risk of coronary heart disease (39). These studies suggest that Grb2 and HSPA8 proteins might play a major role in CAD. Namely, Grb2 contributes to atherosclerotic lesion formation, while basal expression of HSPA8 contributes in reducing the risk of CAD.

Functional enrichment analysis of DEGs belonging to crucial modules showed that DEGs in CAD are mainly involved in protein synthesis, cell apoptosis and signal transduction. Analysis of the related KEGG pathways identified a number of signaling pathways, such as the chemokine signaling pathway. Notably, chemokines and chemokine receptors were reported as important mediators in atherogenesis (40), not only by directing leukocytes into the vessel wall, which is a crucial feature of all stages of atherosclerosis, but also by activating these cells within the atherosclerotic lesion (41). Consequently, the chemokine pathway may represent an important therapeutic target in CAD. In addition, two additional processes with a high RA in our study, focal adhesion (42) and actin regulation (43), were previously reported to be involved in coronary heart disease and the development of atheroma. Briefly, integrin activation can lead to the formation of multi-protein focal adhesion signaling complexes and activate the non-receptor tyrosine kinases focal adhension kinase (FAK) and c-Src (42); regulation of the actin cytoskeletal organization may also contribute to the differential roles of the Rho kinase (ROCK) isoforms in contractility. The RhoA/ROCK signaling pathway is now well recognized as a mediator of vascular smooth muscle contraction in response to vasoconstrictors, by inhibiting myosin phosphatase (MLCP) activity and increasing the phosphorylation of the myosin light chain (43).

$U B C$ was the gene with the highest betweenness centrality in the topological analysis of PPIs. It connected two modules of grade I and III, which were associated with cell activity and signal transduction respectively, but it did not belong to any of the crucial modules. $U B C$ is one of two stress-inducible polyubiquitin genes in mammals, which contributes, along with constitutive UbA genes, in maintaining ubiquitin (Ub) levels during cell proliferation and stress (44). We therefore hypothesize that this gene might act as a connective component for different factors involved in CAD.

In summary, genes differentially expressed in CAD, their potential functions and relevant pathways were identified in this study. The genes $U B C, G R B 2$ and HSPA 8 can be considered as critical genes for $\mathrm{CAD}$, with potential to be used in gene diagnosis and therapy. Moreover, our study suggested that the chemokine pathway might play a vital role in the development of $\mathrm{CAD}$, although further experimental validation is needed to confirm this hypothesis.

\section{References}

1. Eckel RH: Obesity and heart disease: a statement for healthcare professionals from the Nutrition Committee, American Heart Association. Circulation 96: 3248-3250, 1997.

2. Lehto HR, Lehto S, Havulinna AS and Salomaa V: Does the clinical spectrum of incident cardiovascular disease differ between men and women? Eur J Prev Cardiol: March 12, 2013 (Epub ahead of print).

3. Mosca L, Hammond G, Mochari-Greenberger H, Towfighi A, Albert MA, et al: Fifteen-year trends in awareness of heart disease in women: results of a 2012 American Heart Association National Survey. Circulation 127: 1254-1263, 2013.

4. Gheorghiade M, Flaherty JD, Fonarow GC, et al: Coronary artery disease, coronary revascularization, and outcomes in chronic advanced systolic heart failure. Int J Cardiol 151: 69-75, 2011.

5. Bekwelem W, Lutsey PL, Loehr LR, et al: White blood cell count, C-reactive protein, and incident heart failure in the Atherosclerosis Risk in Communities (ARIC) Study. Ann Epidemiol 21: 739-748, 2011.

6. Kwong JS, Leithäuser B, Park JW and Yu CM: Diagnostic value of magnetocardiography in coronary artery disease and cardiac arrhythmias: a review of clinical data. Int J Cardiol 167: 1835-1842, 2013.

7. Alderman EL, Bourassa MG, Cohen LS, et al: Ten-year follow-up of survival and myocardial infarction in the randomized Coronary Artery Surgery Study. Circulation 82: 1629-1646, 1990.

8. Varnauskas E: Twelve-year follow-up of survival in the randomized European Coronary Surgery Study. N Engl J Med 319: 332-337, 1988.

9. The Veterans Administration Coronary Artery Bypass Surgery Cooperative Study Group: Eleven-year survival in the Veterans Administration randomized trial of coronary bypass surgery for stable angina. N Engl J Med 311: 1339-1339, 1984.

10. Califf RM, Harrell FE Jr, Lee KL, et al: The evolution of medical and surgical therapy for coronary artery disease. A 15-year perspective. JAMA 261: 2077-2086, 1989.

11. Gersh BJ, Califf RM, Loop FD, Akins CW, Pryor DB and Takaro TC: Coronary bypass surgery in chronic stable angina. Circulation 79: I46- I59, 1989.

12. Booth J, Clayton T, Pepper J, et al: Randomized, controlled trial of coronary artery bypass surgery versus percutaneous coronary intervention in patients with multivessel coronary artery disease: six-year follow-up from the Stent or Surgery Trial (SoS). Circulation 118: 381-388, 2008.

13. Mark DB, Nelson CL, Califf RM, et al: Continuing evolution of therapy for coronary artery disease. Initial results from the era of coronary angioplasty. Circulation 89: 2015-2025, 1994.

14. Hinkel R, Trenkwalder T and Kupatt C: Gene therapy for ischemic heart disease. Expert Opin on Biol Ther 11: 723-737, 2011.

15. Sinnaeve PR, Donahue MP, Grass P, et al: Gene expression patterns in peripheral blood correlate with the extent of coronary artery disease. PloS One 4: e7037, 2009.

16. Edgar R, Domrachev $M$ and Lash AE: Gene Expression Omnibus: NCBI gene expression and hybridization array data repository. Nucleic Acids Res 30: 207-210, 2002.

17. Davis S and Meltzer PS: GEOquery: a bridge between the Gene Expression Omnibus (GEO) and BioConductor. Bioinformatics 23: 1846-1847, 2007.

18. Saeed A, Sharov V, White J, et al: TM4: a free, open-source system for microarray data management and analysis. Biotechniques 34 : 374-378, 2003.

19. Clements M, van Someren EP, Knijnenburg TA and Reinders MJ: Integration of known transcription factor binding site information and gene expression data to advance from co-expression to co-regulation. Genomics Proteomics Bioinformatics 5: 86-101, 2007.

20. Jensen LJ, Kuhn M, Stark M, et al: STRING 8 - a global view on proteins and their functional interactions in 630 organisms. Nucleic Acids Res 37: D412-D416, 2009.

21. Shannon P, Markiel A, Ozier O, et al: Cytoscape: a software environment for integrated models of biomolecular interaction networks. Genome Res 13: 2498-2504, 2003.

22. Ideker T, Ozier O, Schwikowski B and Siegel AF: Discovering regulatory and signalling circuits in molecular interaction networks. Bioinformatics 18 (Suppl 1): S233-S240, 2002.

23. Kohl M, Wiese S and Warscheid B: Cytoscape: software for visualization and analysis of biological networks. Methods Mol Biol 696: 291-303, 2011. 
24. Huang DW, Sherman BT and Lempicki RA: Systematic and integrative analysis of large gene lists using DAVID bioinformatics resources. Nat Protoc 4: 44-57, 2009.

25. Huang DW, Sherman BT and Lempicki RA: Bioinformatics enrichment tools: paths toward the comprehensive functional analysis of large gene lists. Nucleic Acids Res 37: 1-13, 2009.

26. Assenov Y, Ramírez F, Schelhorn S-E, Lengauer T and Albrecht M: Computing topological parameters of biological networks. Bioinformatics 24: 282-284, 2008.

27. Newman ME: A measure of betweenness centrality based on random walks. Soc Netw 27: 39-54, 2005.

28. Lehmann KA and Kaufmann M: Decentralized algorithms for evaluating centrality in complex networks. Technical Report of the Wilhelm-Schickard-Institut, WSI-2003-10, ISSN 0946-3852, October 2003.

29. Szklarczyk D, Franceschini A, Kuhn M, et al: The STRING database in 2011: functional interaction networks of proteins, globally integrated and scored. Nucleic Acids Res 39: D561-D568, 2011.

30. Giubellino A: GRB2 Signaling as a molecular target for cancer. In: Cell Signaling \& Molecular Targets in Cancer. Springer, New York, NY, pp1-22, 2012.

31. Ideker T and Krogan NJ: Differential network biology. Molecular Syst Biol 8: 565, 2012.

32. Abe J, Manabe I, Aikawa M and Aikawa E: Cardiovascular Inflammation 2012: Reactive Oxygen Species, SUMOylation, and Biomarkers in Cardiovascular Inflammation. Int J Inflam 2013 953463, 2013.

33. Zhang S, Weinheimer C, Courtois $\mathrm{M}$, et al: The role of the Grb2-p38 MAPK signaling pathway in cardiac hypertrophy and fibrosis. J Clin Invest 111: 833-841, 2003.

34. Gutkind JS: Regulation of mitogen-activated protein kinase signaling networks by $\mathrm{G}$ protein-coupled receptors. Sci STKE 2000: re1, 2000.
35. Muslin AJ: MAPK signalling in cardiovascular health and disease: molecular mechanisms and therapeutic targets. Clin Sci 115: 203-218, 2008.

36. Daugaard M, Rohde M and Jäättelä $M$ : The heat shock protein 70 family: Highly homologous proteins with overlapping and distinct functions. FEBS Lett 581: 3702-3710, 2007.

37. Chong KY, Lai CC, Lille S, Chang C and Su CY: Stable overexpression of the constitutive form of heat shock protein 70 confers oxidative protection. J Mol Cell Cardiol 30: 599-608, 1998.

38. Su CY, Chong KY, Owen OE, Dillmann WH, Chang C and Lai CC: Constitutive and inducible hsp70s are involved in oxidative resistance evoked by heat shock or ethanol. J Mol Cell Cardiol 30: 587-598, 1998.

39. He M, Guo H, Yang X, et al: Genetic variations in HSPA8 gene associated with coronary heart disease risk in a Chinese population. PloS One 5: e9684, 2010.

40. Waehre T, Damås JK, Gullestad L, et al: Hydroxymethylglutaryl coenzyme a reductase inhibitors down-regulate chemokines and chemokine receptors in patients with coronary artery disease. J Am Coll Cardiol 41: 1460-1467, 2003.

41. Reape TJ and Groot PH: Chemokines and atherosclerosis. Atherosclerosis 147: 213-225, 1999.

42. Mack CP: Signaling mechanisms that regulate smooth muscle cell differentiation. Arterioscler Thromb Vascular Biol 31: 1495-1505, 2011

43. Wang Y, Zheng XR, Riddick N, et al: ROCK isoform regulation of myosin phosphatase and contractility in vascular smooth muscle cells. Circ Res 104: 531-540, 2009.

44. Ryu K-Y, Maehr R, Gilchrist CA, et al: The mouse polyubiquitin gene $\mathrm{UbC}$ is essential for fetal liver development, cell-cycle progression and stress tolerance. EMBO J 26: 2693-2706, 2007. 